\title{
Planning and Establishing On-Farm Field Trials ${ }^{1}$
}

\author{
Marcelo Wallau, Esteban Rios, and Ann Blount ${ }^{2}$
}

\section{Introduction}

Demonstration plots are one of the oldest and most effective forms of agricultural Extension education-they incorporate "learning by seeing" and "learning by doing" (Leake 1915; Hancock 1997). They provide a great opportunity to test new techniques and technologies "on farmers' fields, and under farmers' conditions and management” (Hancock 1997). They also offer a chance for farmers and recently hired Extension agents to learn about and engage in new management practices. In most cases, demonstration trials are problem-driven and meant to showcase solutions from a local perspective in a proof of concept. In other cases, demonstration plots are organized in partnership with Extension specialists and research faculty, and the demonstrations are part of a research (hypothesis-driven) trial designed to test specific hypotheses. In both cases, nothing is more frustrating than a failed trial. Unorganized, poorly kept, or badly managed demonstration sites undermine the technique or technology presented and diminish the Extension agent's credibility. Planning and preparation can increase chances of successful outcomes from demonstration plots. This document focuses on guiding the successful establishment of demonstration trials and is targeted to county, regional, and state specialized Extension faculty who aim to develop on-farm research and demonstration sites as part of their programs.

\section{Planning a Demonstration Site Defining Objectives}

The first step of any successful demonstration trial is defining the objectives:

- What will be tested/shown?

- Will this demonstration plot also involve a hypothesisdriven research trial?

- Will data be collected?

- Will this trial be replicated at other locations?

- When will this trial be showcased?

- Who is the target audience?

- What are the expected outcomes?

In general, the idea for a demonstration plot starts with a need from the community (e.g., on-farm cultivar tests, plant spacing and/or planting date trial, etc.), or a desire of an Extension agent or specialist to showcase a new technique or technology. The first case is straightforward, especially because there is direct community involvement and interest, and the demonstration typically involves proven techniques. It is a matter of following production guidelines. In the latter case, careful planning is essential, especially when proposing a technique or technology that has not yet been tested in the target environment's specific conditions. Think of aspects that may cause the trial to fail and other challenges that would hinder application or

1. This document is SS-AGR-447, one of a series of the Agronomy Department, UF/IFAS Extension. Original publication date January 2021. Visit the EDIS website at https://edis.ifas.ufl.edu for the currently supported version of this publication.

2. Marcelo Wallau, assistant professor, forage Extension specialist, Agronomy Department; Esteban Rios, assistant professor, forage breeding and genetics, Agronomy Department; and Ann Blount, professor, forage breeding and genetics, Agronomy Department, UF/IFAS North Florida Research and Education Center; UF/IFAS Extension, Gainesville, FL 32611.

The Institute of Food and Agricultural Sciences (IFAS) is an Equal Opportunity Institution authorized to provide research, educational information and other services

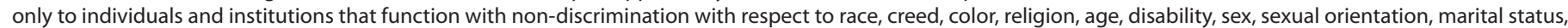

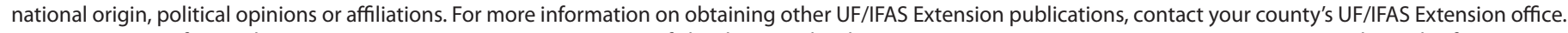
U.S. Department of Agriculture, UF/IFAS Extension Service, University of Florida, IFAS, Florida A \& M University Cooperative Extension Program, and Boards of County Commissioners Cooperating. Nick T. Place, dean for UF/IFAS Extension. 
reduce interest. A good exercise is to consider why farmers are not already adopting the proposed practice. There might be several reasons, such as lack of access to markets for a new product or limited availability of the proposed input. A good strategy is to involve your collaborator or advisory committee in the planning process and test your ideas against their experience.

Note that the demonstration trial may not work or show treatment differences in the first year. Lack of experience and tools as well as certain technologies' delayed impacts can pose challenges to broader changes in the current agricultural system. For example, conservation agriculture requires specialized equipment and may take several cropping seasons to improve soil quality before major results become apparent. In this case, persistence is important, and conclusions should not be drawn in the first year.

\section{Choosing the Right Collaborator}

Selecting suitable collaborators is crucial to the success of on-farm demonstrations and the effective dissemination of information. Ideally, you need to work with prominent and progressive farmers: early adopters willing to try new technologies or techniques. Collaborators need to be fairly representative of the larger target audience so their experiences will translate well to the larger group. Choose reliable and honest collaborators who are respected leaders in the community and will follow through with the trial protocol. Even though collaborators may be interested in the proposed project, they might see the trial as a burden and in the way of their normal farm activities. Needs, roles, and responsibilities must be very clear from the beginning to avoid issues. If you cannot find an acceptable collaborator for the project, consider establishing the demonstration trial at an Experimental Station or Research Unit, where proper machinery is available, and the staff have experience conducting trials.

\section{Choosing the Demonstration Site}

There are two crucial questions to consider during site selection.

1. Is the site suitable for the proposed demonstration trial?

2. Is the site easily accessible?

Select practices and species or cultivars that are recommended and suitable for the chosen site. Consider soil and climatic conditions and check to see if they are suited to the practices proposed or plant species to be established. Meet with the collaborator and investigate the history of the area. Identify which crops were previously grown. Check for possible residual nutrient or chemical effects from previous management practices, and find out about known issues with pests, weeds, and diseases. Consider how these issues might affect your findings. In terms of accessibility, think about your workload, required number of visits to the site during the growing season, and level of access to the site (e.g., easy, practical, close to the office, en route to another location you need to visit, etc.). If visiting and maintaining the demonstration site takes more time than you can allocate, the site may fall into disrepair. Consider how clientele can access the site for an educational program or other activity (e.g., can a van or small vehicle access the site? Does the site have infrastructure for a field day? How many people can the place accommodate?).

\section{Defining Treatments, Trial Design, and Treatment Application}

After defining the objectives of the trial, you need to define the treatments and design the experiment. The questions to be addressed help to define experimental and treatment designs. The more complex those questions are, the more complex the design is likely to become. Generally, it is advisable to make it simple, so fewer things can go wrong. A single-factor treatment design is simplest because it involves only multiple levels for a single variable (e.g., different cultivars or different fertilization rates). A standard (control) treatment should be included to serve as a comparison baseline. Normally, the control is a current practice or common cultivar. Once treatments are defined and applied, there should be no changes to the trial and the protocol must be maintained throughout the course of the trial. Generally, for agricultural research, a randomized complete block design with four blocks is recommended (Figure 1; see Davis et al. [2017] for more detailed information on experimental design for on-farm trials). The advantage of replicated trials is to account for environmental variation and isolate the field effect (error) from the actual treatment effect. It is highly recommended that treatments be randomized in each block independently.

Most on-farm demonstrations (proof-of-concept) trials are not replicated, leading to biased and weak conclusions. Those trials can still be useful as an example of a technique or technology and for observation. We do not recommend implementing unreplicated demonstration plots if the goal is to collect data or draw any major conclusions (the exception being if the plots are part of a larger-scale study with multiple locations and are statistically evaluated, as in the case of a stability analysis for new cultivars). Field variation can be confounded with treatment effect. Without 
replications, there is no way to separate the differences due to uncontrolled variation (error) or the treatment. From a statistical standpoint, replicates are necessary to analyze the variance in order to draw unbiased conclusions from the fieldwork with a certain level of confidence (Nafziger 1984). Large plot size does not compensate for lack of replication or reduce plot-to-plot variability, nor does increasing the number of sampling units within an experimental unit. Neither approach can be used to assess within-treatment variability. An alternative to the use of single plots within a site is to use multiple sites (e.g., multiple farmers or multiple counties) and consider each location as a block with all treatments present. With this approach, we cannot analyze the location effect (e.g., which hybrid or variety performs better in each location), but we can assess the overall treatment effect across locations. When doing this, it is important to coordinate with other farmers and agents to ensure that the same protocol is followed, with similar planting dates and management practices. Ideally, the same demonstration should be planted at a minimum of four locations.

Side-by-side (non-randomized; demonstration block on Figure 1) demonstrations are very useful to show a sequence of treatments or cultivars. This setup is frequently used for variety trials where there are several cultivars of one species or several cultivars/hybrids from one company. This approach, however, can be influenced by field variation, especially if there are no randomized replications. This block should not be included in the statistical analysis, because the error is not randomly distributed among treatments. Imagine an experimental area with a slight slope: corn hybrids on the lower side of the slope are likely to have more water available and accumulate nutrients leached or run off from the higher side of the field. Thus, the increased productivity of those hybrids could be due to favorable environmental conditions instead of true genetic superiority. Blocks should always be perpendicular to the direction of potentially confounding field variation (Figure 1). Other potential sources of field variation include soil type, shade from trees along field edges or fencerows, irrigation system variations, wet spots, previous peanut windrows, old hay feeding sites, old driveways, etc.

When working with multiple factors (e.g., different species planted under different land preparation methods), it is possible to arrange treatments to create what is called a "split-plot" arrangement. For example, consider that we want to investigate or demonstrate the effect of two land preparation techniques (e.g., till vs. no-till) on the production of cool-season forages (ryegrass vs. oat). We have a $2 \times 2$ factorial for a total of four treatments (Figure 2 ). Ideally, we would have four plots per block, each one with a combination of land preparation and cool-season forage species. Because land preparation requires large equipment and more effort, it is possible to consider this as the whole plot treatment. Each whole plot can then be split in half to overlay the cool-season species ("subplot"). The sub plot should be the factor in which we are most interested.

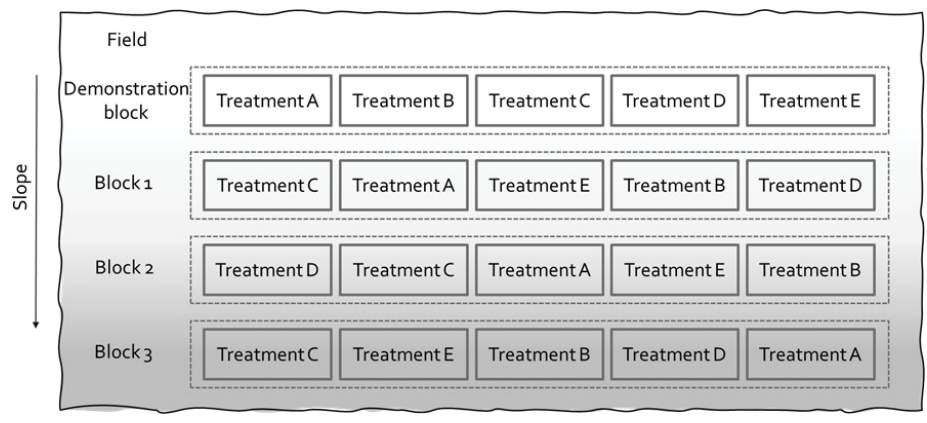

Figure 1. Example of a trial with five treatments and four blocks: one demonstration-only block and three for data collection. All blocks are distributed at the same level in the slope to reduce confounding effects of the slope on the treatments.

Credits: UF/IFAS

Block

\begin{tabular}{l|l|} 
Plot: tilled & Plot: no-till \\
\hline Sub-plot: oat & Sub-plot: ryegrass \\
\hline Sub-plot: ryegrass & \\
\hline
\end{tabular}

Figure 2. Example of a split-plot arrangement where the whole plot is land preparation (tilled ground vs. no-till) and the sub plot is coolseason forage species (oat or ryegrass).

Credits: UF/IFAS

A plot is the area where individual treatments are applied (experimental unit). Plot size can vary from square feet to a few acres. Large plots are not necessarily better; they require extra work and resources. If the trial is well designed and implemented, plots can be minimal in size; however, the type of material or technique presented will affect the necessary plot size. The amount of land needed to demonstrate the biological effect and to minimize the influence of other treatments or borders in the sampling area determines minimal plot size. The available equipment and lay of the land often determine actual plot size. For example, if we have a $6 \mathrm{ft}$ no-till drill to plant cool-season forages, then our plots will be $6 \mathrm{ft}$ wide or multiples of 6 . Consider other equipment needed for maintenance (such as sprayers) and harvesting as well. 


\section{Written Protocol}

Always have a written protocol before establishing a field trial. This document should contain information on the problem being addressed (the problem statement or objective), the expected outcomes and benefits, the treatments to be applied, and especially your expectations of the farmer (i.e., define their roles in the project). Include a detailed materials and methods section with information related to treatment application, sampling procedures, and an expected maintenance and sampling schedule. Standardized treatment application and sampling procedures are extremely important, especially when dealing with multiple collaborators and/or when the collaborator has multiple employees/family members who will be involved with the process. The protocol should serve as a guide so there is no doubt about what must be done and when and how to do it. This must be clear from the beginning, so all parties involved know what to expect and know their roles.

\section{Establishing and Maintaining the Demonstration Site}

Trial establishment and maintenance depend on the crop or practice being investigated. As a rule of thumb, determine a baseline by characterizing the scenario. For agricultural trials, take soil or substrate samples, and amend or fertilize as required for the crop. Land preparation should be planned in advance, including liming and herbicide applications. Many trials fail due to poor fertility and high weed pressure. Follow recommended practices for the establishment and maintenance of the crop to enhance chances of success. Once the area has been prepared, take time in the field to lay out the plots, mark, and label them. A well-designed and laid out experimental area facilitates treatment application and comparison, and allows a better-organized field day. Equipment calibration-such as that for planters, spreaders, and sprayers-is essential to treatment applications and trial maintenance. Know your equipment and have a plan beforehand on how to calibrate. Be sure you have all of the necessary tools and information to calibrate the implements. Lack of preparation wastes the collaborator's time, looks unprofessional, and leads to mistakes. See Forage Planting and Establishment Methods on Prepared Seedbed (https://edis.ifas.ufl.edu/ag107) and Calibrating Forage Seeding Equipment (https://edis.ifas. ufl.edu/ag158) for more information. Plot maintenance depends on the treatments applied. In general, pay attention to fertilization and pest/weed control schedules and visit the site frequently to monitor for pests, diseases, or other stresses. These practices help to avoid any confounding effect (e.g., yield-limiting problems) on your treatments.

\section{Collecting Information}

Keep a detailed log of all procedures and management practices throughout the trial. Define the baselines and collect weather information. A mobile weather station can be very helpful and fairly inexpensive. At the very least, make sure you have a rain gauge and thermometer to register minimum and maximum temperatures. Regional weather data are also available on the Florida Automated Weather Network website (https://fawn.ifas.ufl.edu/). Take pictures and videos of the evolution of the trial. This will help with preparing and disseminating any informational or creative material later. The field day is only one day-it may not convey the big picture of the trial (Nafziger 1984).

The quality of the information obtained from the trial is only as good as the rigor applied. Follow all sampling guidelines and schedules to obtain reliable data. If not replicated, protocols are very limited. As previously mentioned, values collected should not be used to draw any major conclusion. Use standardized units, such as dry matter production or supplementation per unit of body weight. Also, be careful when extrapolating results from small plots to large areas: the numbers can be deceiving and might have been influenced by other factors (such as border effects or "extra care" for the small parcels). It is advised to work with a state specialist or research faculty member to make sure the experimental protocol is adequate, and the data collected are reliable and can be analyzed. For help designing the trial and analyzing data, UF/IFAS offers statistical consulting services (https://forms.stat.ufl.edu/forms/consulting/). Consult the statistician before establishing the trial, because little can be done about the design afterwards.

\section{Planning a Field Day}

Preparation for a field day takes a lot of effort and involvement. Nevertheless, it is the time to showcase all your hard work during the growing season. Think specifically of the trial and prepare the area beforehand. Mow, clean, spray, or till the alleys. Create clear signs with the treatment description and posters or handouts with management information and main results. A well-presented trial has more appeal than a sloppy field. It will help the audience to focus on the actual treatments, instead of on which weeds are growing, and enable you to present the trial more efficiently. Remember to have a checklist-it will help you keep track of all the activities and materials. 


\section{Common Failures}

\section{Common Field Failures}

The most common failure in field trials is poor establishment and/or treatment application. Site preparation must be done in advance; you need to check with your collaborator well before the planting date. Treatment application needs to be precise and follow the proposed protocol. If the trial fails from the start, there is nothing that can be done to remediate. The second major problem is lack of maintenance. The trial must be checked frequently. You cannot plant and only return to the site for the field day. It is very common for the agent to expect the farmer to check the trial, while the farmer waits for the agent to visit the site. Maintain good communication with your collaborators, especially when working with other agents or volunteers in your office. Planning and dedication are essential.

\section{Working with Collaborators}

If the farmer has no vested interest in the technique or technology, the demonstration has a greater likelihood of failure. If working with a skeptical famer to whom you want to prove a point, it is important to visit the demonstration periodically to make sure the protocol is being followed and things are as expected. A broken fence or a mistake during spraying can jeopardize your demonstration.

There are many signs to "back off." That is best done before the trial starts, so no resources are wasted, and relationships are not injured. If a farmer is not willing to cooperate or is not convinced of what is going to be done, or if success depends on more dedication than they are willing to give, it might be best not to conduct the trial. Questions such as "Is this really necessary," "Why can't I just turn my cows loose here," or "Do I really need to wait that long" are signs that there is not a good understanding of the necessity of respecting the demonstration protocol. In this case, the demands of the demonstration may be interfering with the normal farm schedule or adding to the farmer's workload. The farmer may not want to help or may not see the purpose and need for the protocol or demonstration. Once the trial is established, frequently checking it and communicating with the farmer can prevent some of these issues, or at least give you time to draft an alternative strategy if plans change.

\section{Final Remarks}

On-farm demonstrations can be fantastic learning tools and a great way to involve farmers in the process. Many factors must be taken into consideration for a successful trial. A well-planned trial increases the likelihood of success and is easier to implement, manage, and report. However, trials require a lot of dedication and investments of time and money. It is important to balance the complexity and involvement of the proposed trial with the expected outcomes. When possible, partner with state specialists and researchers who can provide knowledge and resources (including funding and labor) to improve your trial. Make sure that there is a good understanding of roles and responsibilities to avoid issues later. Demonstration sites can also be used to prepare creative material and publish in trade journals or peer-reviewed outlets.

\section{References}

Davis, R. F., G. H. Harris, P. M. Roberts, and G. E. MacDonald. 2017. "Designing Research and Demonstration Tests for Farmer's Fields." University of Georgia Extension. Bulletin 1177. 8 pp.

Hancock, J. 1997. Extension Education: Conducting Effective Agricultural Demonstrations. University of Kentucky Cooperative Extension Services. Handbook ID-111. 19 pp.

Leake, A. H. 1915. The Means and Methods of Agricultural Education. Cambridge, MA: The Riverside Press. 274 pp.

Nafziger, E. D. 1984. "Use of Demonstration Plots as Extension Tools." Journal of Agronomic Education 13:47-49. 